\title{
Anomalous Nernst and Hall effects in magnetized platinum and palladium
}

\author{
G. Y. Guo,, , 2, * Q. Niu, ${ }^{3,4}$ and N. Nagaosa ${ }^{5,6}$ \\ ${ }^{1}$ Department of Physics, National Taiwan University, Taipei 10617, Taiwan \\ ${ }^{2}$ Graduate Institute of Applied Physics, National Chengchi University, Taipei 11605, Taiwan \\ ${ }^{3}$ International Center for Quantum Materials and Collaborative Innovation \\ Center of Quantum Matter, Peking University, Beijing 100871, China \\ ${ }^{4}$ Department of Physics, The University of Texas at Austin, Austin, Texas 78712, USA \\ ${ }^{5}$ Department of Applied Physics, University of Tokyo, Tokyo 113-8656, Japan \\ ${ }^{6}$ RIKEN Center for Emergent Matter Science (CEMS), Wako, Saitama 351-0198, Japan
}

(Dated: October 1, 2018)

\begin{abstract}
We study the anomalous Nernst effect (ANE) and anomalous Hall effect (AHE) in proximityinduced ferromagnetic palladium and platinum which is widely used in spintronics, within the Berry phase formalism based on the relativistic band structure calculations. We find that both the anomalous Hall $\left(\sigma_{x y}^{A}\right)$ and Nernst $\left(\alpha_{x y}^{A}\right)$ conductivities can be related to the spin Hall conductivity $\left(\sigma_{x y}^{S}\right)$ and band exchange-splitting $\left(\Delta_{e x}\right)$ by relations $\sigma_{x y}^{A}=\Delta_{e x} \frac{e}{\hbar} \sigma_{x y}^{S}\left(E_{F}\right)^{\prime}$ and $\alpha_{x y}^{A}=-\frac{\pi^{2}}{3} \frac{k_{B}^{2} T \Delta_{e x}}{\hbar} \sigma_{x y}^{s}(\mu)^{\prime \prime}$, respectively. In particular, these relations would predict that the $\sigma_{x y}^{A}$ in the magnetized $\mathrm{Pt}(\mathrm{Pd})$ would be positive (negative) since the $\sigma_{x y}^{S}\left(E_{F}\right)^{\prime}$ is positive (negative). Furthermore, both $\sigma_{x y}^{A}$ and $\alpha_{x y}^{A}$ are approximately proportional to the induced spin magnetic moment $\left(m_{s}\right)$ because the $\Delta_{e x}$ is a linear function of $m_{s}$. Using the reported $m_{s}$ in the magnetized $\mathrm{Pt}$ and $\mathrm{Pd}$, we predict that the intrinsic anomalous Nernst conductivity (ANC) in the magnetic platinum and palladium would be gigantic, being up to ten times larger than, e.g., iron, while the intrinsic anomalous Hall conductivity (AHC) would also be significant.
\end{abstract}

PACS numbers: 72.15.Gd, 72.15.Jf, 72.25.Ba, 75.76. $+\mathrm{j}$

\section{INTRODUCTION}

Spin transport electronics (spintronics) has recently attracted enormous attention mainly because of its promising applications in information storage and processing and other electronic technologies [1, 2]. Spin current generation, detection and manipulation are three key issues in the emerging spintronics. Large intrinsic spin Hall effect (SHE) in platinum has recently been predicted [3] and observed (see Refs. 4 and 5 and references therein). In the SHE, a transverse spin current is generated in response to an electric field in a metal with relativistic electron interaction. The SHE enables us to generate and control spin current without magnetic field or magnetic materials, which would be an important step for spintronics. Furthermore, in the inverse spin Hall effect, a transverse voltage drop arises due to the spin current [6, 7], and this allows us to detect spin current by measuring the Hall voltage. Therefore, platinum has been widely used as a spin current generator and detector in recent spin current experiments, such as spin Seebeck effect [8], spin pumping [9] and spin Hall switching [10], and plays a unique role in recent developments in spintronics.

Platinum is an enhanced paramagnet because its $5 d$-band is partially filled with a large density of states (DOS) at the Fermi level $\left(E_{F}\right)\left[N\left(E_{F}\right)=\sim 1.74\right.$ states/eV/spin]. Consequently, it could become ferromagnetic with a significant spin magnetic moment when placed next to a ferromagnetic metal [11, 12] or in lowdimensional structures such as an atomic bilayer on silver
(001) surface 13] or a freestanding atomic chain 14, 15]. Indeed, platinum was reported to possess a magnetic moment as large as $\sim 0.2$ and $\sim 0.5 \mu_{B} /$ atom in $\mathrm{Ni} / \mathrm{Pt}$ and $\mathrm{Fe} / \mathrm{Pt}$ multilayers [11, 12], respectively. In a ferromagnetic metal, a transverse charge current would be generated in response to an electric field due to relativistic spin-orbit coupling (SOC), an effect known as the anomalous Hall effect (AHE) [16], discovered by Hall[17] long ago. Since the AHE is another archetypal spin-related transport phenomenon 16 and the SOC strength in $\mathrm{Pt}$ is large, it would be interesting to study the AHE in the proximity-induced ferromagnetic platinum. Furthermore, as pointed out in Ref. 18, the fact that the Hall voltage could be generated by both the AHE and inverse SHE in the magnetized platinum, might complicate the detection of the pure spin current and also related phenomena using platinum. Therefore, it is important to understand the transport and magnetic properties of the magentized platinum.

In a ferromagnet, the Hall voltage could also arise when a thermal gradient instead of an electric field, is applied. This phenomenon, again due to the relativistic SOC, is refered to as the anomalous Nernst effect (ANE) 19]. Interestingly, the ANE could be used as a probe of the vortex phase in type II superconductors [20] and has been receiving considerable attention in recent years. 21 28] In this context, it would be interesting to study the ANE in the proximity-induced ferromagnetic platinum. On the other hand, spin Seebeck effect (another thermal phenomenon), which refers to the generation of a spin-motive 
force in a ferromagnet by a temperature gradient, has recently attracted considerable attention [8, 29, 30]. Again, this effect is usually measured as a transverse voltage in a nonmagnetic metal such as $\mathrm{Pt}$ in contact with the ferromagnet via the inverse SHE[8]. Clearly, if the metal is magnetized due to the magnetic proximity effect, the ANE would contribute to the measured Hall voltage too. In this connection, it is imperative to understand the ANE in the magnetized platinum.

Palladium is isoelectronic to platinum and thus has an electronic structure similar to that of $\mathrm{Pt}$ except a smaller SOC strength (see, e.g., Refs. 3 and 31 and references therein). For example, like $\mathrm{Pt}, \mathrm{Pd}$ also has a large intrinsic spin Hall conductivity (SHC) [31] and is a highly enhanced paramagnetic metal with a large $N\left(E_{F}\right)=\sim 2.69$ states $/ \mathrm{eV} / \mathrm{spin}$. In fact, palladium possesses the largest paramagnetic susceptibility of $567 \times$ $10^{-6} \mathrm{emu} /$ mole among the nonmagnetic metals 32 and is usually considered to be nearly ferromagnetic. It could become ferromagnetic when placed next to a ferromagnetic metal [33, 34] or fabricated as an atomic bilayer on silver (001) surface 13] or a freestanding atomic chain [15]. Recently, the AHE was observed in the Pd film on an yttrium iron garnet (YIG). 35] Surprisingly, it was reported that the intrinsic anomalous Hall conductivity (AHC) in the Pd film on the YIG layer has a sign opposite to that for the Pt/YIG bilayer. 35. This indicates that the AHC in a magnetized nonmagnetic metal does not simply scale with the SOC strength. One would then ask what determines the AHC in the magnetized metals.

In this paper, therefore, we study the AHE and ANE in the proximity-induced ferromagnetic platinum and palladium within the Berry phase formalism [36] based on firstprinciple relativistic band structure calculations. We also perform analytic calculations to identify possible relations between the SHC in an nonmagnetic metal and the AHC in the corresponding magnetized metal. The rest of this paper is organized as follows. In the next section, we briefly describe the Berry phase formalism for calculating the $\mathrm{AHC}$ and $\mathrm{ANC}$ as well as the computational details. In Sec. III, the calculated AHC and ANC will be presented. Finally, the conclusions drawn from this work will be summarized in Sec. IV.

\section{THEORY AND COMPUTATIONAL DETAILS}

The anomalous Hall conductivity and anomalous Nernst conductivity (ANC) are calculated by using the Berry-phase formalism 36]. Within this Berry-phase formalism, the AHC is simply given as a Brillouin zone (BZ) integration of the Berry curvature for all the occupied bands,

$$
\begin{array}{r}
\sigma_{x y}^{A}=-\frac{e^{2}}{\hbar} \sum_{n} \int_{B Z} \frac{d \mathbf{k}}{(2 \pi)^{3}} f_{\mathbf{k} n} \Omega_{n}^{z}(\mathbf{k}), \\
\Omega_{n}^{z}(\mathbf{k})=-\sum_{n^{\prime} \neq n} \frac{2 \operatorname{Im}\left[\left\langle\mathbf{k} n\left|v_{x}\right| \mathbf{k} n^{\prime}\right\rangle\left\langle\mathbf{k} n^{\prime}\left|v_{y}\right| \mathbf{k} n\right\rangle\right]}{\left(\epsilon_{\mathbf{k} n}-\epsilon_{\mathbf{k} n^{\prime}}\right)^{2}},
\end{array}
$$

where $f_{\mathbf{k} n}$ and $\Omega_{n}^{z}$ are the Fermi distribution function and the Berry curvature for the $n$th band at $\mathbf{k}$, respectively. 37. Similarly, the ANC can be written as

$$
\begin{array}{r}
\alpha_{x y}^{A}=\frac{1}{T} \frac{e}{\hbar} \sum_{n} \int_{B Z} \frac{d \mathbf{k}}{(2 \pi)^{3}} f_{\mathbf{k} n} \Omega_{n}^{z}(\mathbf{k}) \\
\times\left[\left(\epsilon_{\mathbf{k} n}-\mu\right) f_{\mathbf{k} n}+k_{B} T \ln \left(1+e^{-\beta\left(\epsilon_{\mathbf{k} n}-\mu\right)}\right)\right],
\end{array}
$$

where $\mu$ is the chemical potential and $k_{B}$ is the Boltzmann constant. 22.

The proximity-induced ferromagnetic platinum and palladium are investigated by the constrained spindensity functional theory with the local density approximation to the exchange-correlation potential. 38. Spin-polarized self-consistent scalar-relativistic electronic structure calculations with the spin magnetic moment fixed to specified values, are performed. Using the resultant self-consistent charge densities, the fully relativistic band structures are then calculated for the AHC and ANC calculations. The highly accurate all-electron fullpotential linearized augmented plane wave (FLAPW) method, as implemented in the WIEN2K code [39], is used. The experimental lattice constants $a=3.92$ and $3.89(\AA)$ are used, respectively, for Pt and Pd. In both cases, the muffin-tin sphere radius $\left(R_{m t}\right)$ of 2.5 a.u. is adopted. The wave function, charge density, and potential are expanded in terms of the spherical harmonics inside the muffin-tin spheres and the cutoff angular moment $\left(L_{\max }\right)$ used is 10,6 and 6 , respectively. The wave function outside the muffin-tin sphere is expanded in terms of the augmented plane waves (APWs) and a large number of APWs (about 70 APWs per atom, i. e., the maximum size of the crystal momentum $K_{\max }=9 / R_{m t}$ ) are included in the present calculations. The tetrahedron method is used for the BZ integration [40]. To obtain accurate ground state properties, a fine $21 \times 21 \times 21$ grid of $11616 k$-points in the first BZ is used. For the AHC and ANC calculations, a very find grid of $258156 k$-points on the magnetic irreducible wedge $(1 / 16 \mathrm{BZ})$ in the $\mathrm{BZ}$ is used. This is equivalent to a large number of $k$-points of $\sim 4000000$ in the full BZ, and corresponds to the division of the $\Gamma X$ line into 70 intervals. Comparison with test calculations with a denser grid of $381915 k$-points (80 divisions of the $\Gamma X$ line) indicates that the calculated AHC and ANC converge to within a few $\%$. 


\section{RESULTS AND DISCUSSION}

The relativistic band structure and also $\mathrm{AHC}\left(\sigma_{x y}^{A}\right)$ as a function of the Fermi energy $\left(E_{F}\right)$ for the magnetized platinum and palladium with the spin magnetic moment $m_{s}=0.1 \mu_{B}$ /atom are displayed in Figs. 1 and 2, respectively. All Kramer-degenerate bands in nonmagnetic platinum (see Fig. 1 in [3]) and palladium (see Fig. 1 in [31]) are now exchange-split due to the induced magnetization in the magnetized $\mathrm{Pt}$ and $\mathrm{Pd}$. This is clearly visible for the $d$-dominated bands [i.e., energy bands below $1.0 \mathrm{eV}$ in Fig. 1(a) or $0.5 \mathrm{eV}$ in Fig. 2(a)] since the ferromagnetism is mainly caused by the exchange interaction among the $d$-electrons. The band spin-splittings are largest in the flat bands of almost pure $d$ character such as the bands around $0.5 \mathrm{eV}(0.3 \mathrm{eV})$ in the vicinity of the W-point in Fig. 1(a) [Fig. 2(a)].

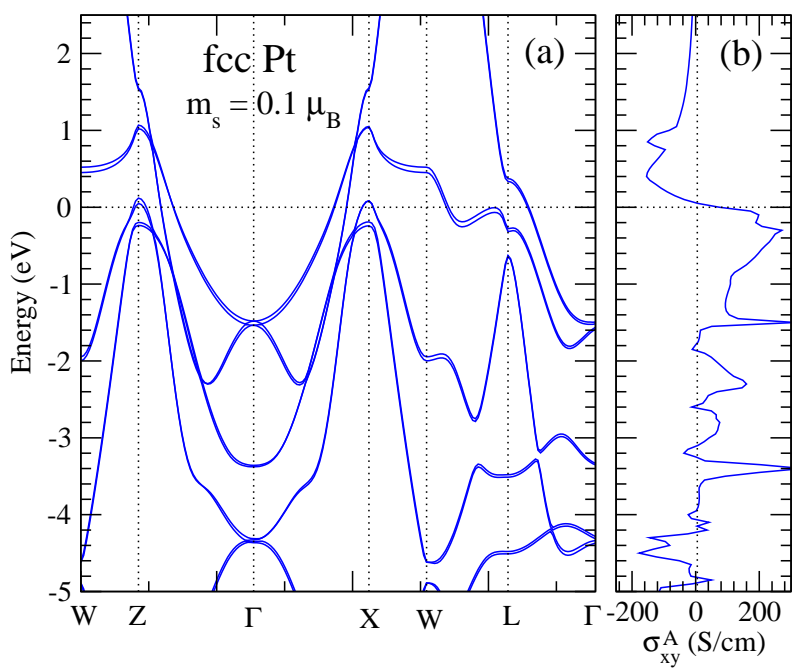

FIG. 1: (Color online) (a) Relativistic band structure and (b) anomalous Hall conductivity (AHC) of the magnetized platinum with a spin magnetic moment of $0.1 \mu_{B}$ /atom. The horizontal dotted line at the zero energy indicates the Fermi level.

\section{Anomalous Hall effect}

Figures 3 and 4 show the calculated AHC and ANC $\left(\alpha_{x y}^{A}\right)$ as well as the exchange splitting $\left(\Delta_{e x}\right)$ as a function of the induced spin magnetic moment $\left(m_{s}\right)$ in platinum and palladium, respectivelty. $\Delta_{e x}$ refers to the splitting of the spin-up and spin-down bands, and we calculate $\Delta_{e x}$ as the spin splitting of the scalar-relativistic bands above the Fermi level at the W-point [Figs. 1(a) and 2(a)]. First of all, it is clear from Figs. 3 and 4 that the calculated $\sigma_{x y}^{A}$ and $\Delta_{e x}$ increase monotonically with $m_{s}$. In fact, $\Delta_{e x}$ is almost perfectly proportional to $m_{s}$, while the amplitude of the $\sigma_{x y}^{A}$ increases linearly with $m_{s}$ for

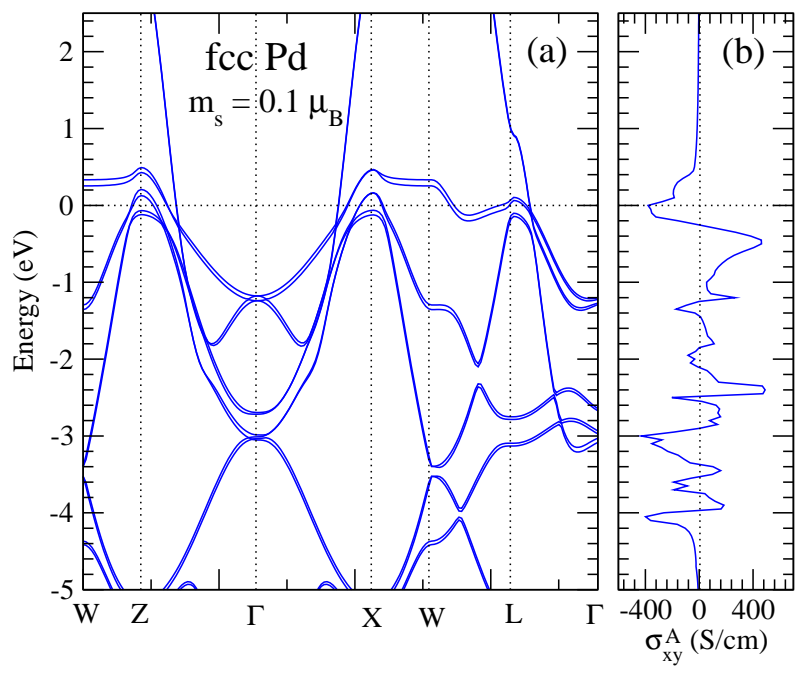

FIG. 2: (Color online) (a) Relativistic band structure and (b) anomalous Hall conductivity of the magnetized palladium with a spin magnetic moment of $0.1 \mu_{B}$ /atom. The horizontal dotted line at the zero energy indicates the Fermi level.

small $m_{s}$ values up to 0.30 and $0.25 \mu_{B}$ /atom for Pt and $\mathrm{Pd}$, respectively.

Secondly, the AHC is large. In particular, the magnitude of the AHC per $\mu_{B}\left(\sigma_{x y}^{A} / m_{s}\right)$ for $m_{s} \leq 0.25$ $\mu_{B} /$ atom in $\mathrm{Pt}$ and $\mathrm{Pd}$ is, respectively, $\sim 790$ and $3500 \mathrm{~S} /\left(\mathrm{cm} \cdot \mu_{B}\right)$, being much larger than that of $\sim 360$ $\mathrm{S} /\left(\mathrm{cm} \cdot \mu_{B}\right)$ in iron 37]. Interestingly, the ratio $\sigma_{x y}^{A} / m_{s}$ for $\mathrm{Pt}$ is smaller than that for $\mathrm{Pd}$, indicating that the $\mathrm{AHC}$ in a proximity-induced ferromagnetic metal is not necessarily correlated with the SOC strength. Thirdly, the sign of the $\mathrm{AHC}$ in $\mathrm{Pt}$ is opposite to that in $\mathrm{Pd}$, being in good agreement with the recent experiments on the Pt/YIG and Pd/YIG bilayers 35.

\section{Correlation between anomalous and spin Hall conductivities}

In order to gain insight into the key factors that determine the $\mathrm{AHC}$ in a magnetized nonmagnetic metal, let us consider the two-current model to connect the conductivities for the different sorts of Hall effects. Within the two-current model approximation, $\sigma_{x y}^{A}$ and $\sigma_{x y}^{S}$ can be written as 41 43] $\sigma_{x y}^{A}(E)=\sigma_{x y}^{\uparrow}(E)+\sigma_{x y}^{\downarrow}(E)$ and $-2 \frac{\hbar}{e} \sigma_{x y}^{S}(E)=\sigma_{x y}^{\uparrow}(E)-\sigma_{x y}^{\downarrow}(E)$, where $\sigma_{x y}^{\uparrow}$ and $\sigma_{x y}^{\downarrow}$ are the spin-up and spin-down Hall conductivities, respectively. In a non-magnetic metal, the spin magnetic moment $m_{s}=0$ and thus, $\sigma_{x y}^{A}=0$. In the magnetized metal, $\sigma_{x y}^{A}(E)=\sigma_{x y}^{\uparrow}\left(E-\frac{1}{2} \Delta_{e x}\right)+\sigma_{x y}^{\downarrow}\left(E+\frac{1}{2} \Delta_{e x}\right) \approx$ $\sigma_{x y}^{\uparrow}(E)-\frac{1}{2} \Delta_{e x} \sigma_{x y}^{\uparrow}(E)^{\prime}+\sigma_{x y}^{\downarrow}(E)+\frac{1}{2} \Delta_{e x} \sigma_{x y}^{\downarrow}(E)^{\prime}$, where $\Delta_{e x}$ is the exchange splitting and is proportional to $m_{s}$, as shown in the inset in Fig. 3(a) and Fig. 4(a). There- 

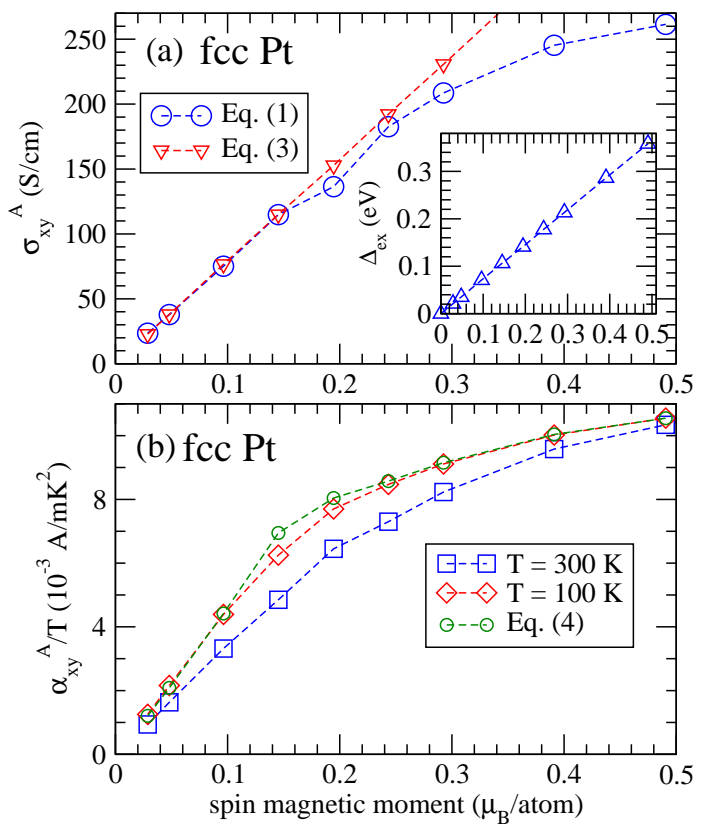

FIG. 3: (Color online) (a) Anomalous Hall conductivity $\left(\sigma_{x y}^{A}\right)$ and (b) anomalous Nernst conductivity $\left(\alpha_{x y}^{A}\right)$ as a function of the induced spin magnetic moment $\left(m_{s}\right)$ in platinum. Exchange splitting $\left(\Delta_{e x}\right)$ is displayed as a function of $m_{s}$ in the inset in (a). In (b), $T$ denotes temperature.

fore, we find

$$
\sigma_{x y}^{A}\left(E_{F}\right) \approx \Delta_{e x} \frac{e}{\hbar} \sigma_{x y}^{S}\left(E_{F}\right)^{\prime} .
$$

Equation (3) tells us that the AHC is proportional to the energy derivative of the spin Hall conductivity $\left[\sigma_{x y}^{S}\right]$ as well as the exchange splitting $\left(\Delta_{e x}\right)$. Interestingly, the SOC strength does not appear explicitly in Eq. (3), in contrary to conventional wisdom. We notice that platinum and palladium have similar AHC-versus-energy $\left[\sigma_{x y}^{A}(E)\right]$ curves which have a prominent peak near the $E_{F}$ (see Fig. 1 in both [3] and [31]). However, the $E_{F}$ falls on the up-hill side of the peak in $\mathrm{Pt}[3]$ but on the down-hill side of the peak in $\mathrm{Pd}[31]$, resulting in the positive $\sigma_{x y}^{S}\left(E_{F}\right)^{\prime}$ for $\mathrm{Pt}$ and negative $\sigma_{x y}^{S}\left(E_{F}\right)^{\prime}$ for $\mathrm{Pd}$. This, together with Eq. (3), naturally explains why both the calculated and observed AHCs in Pt and Pd have opposite signs.

To examine quantitatively the validity of Eq. (3), here we repeat the calculations of the $\mathrm{SHC}$ for $\mathrm{Pt}[3]$ and $\mathrm{Pd}[31]$ but using the more accurate FLAPW method with the same computational details as described already in Sec. II. The calculated SHC for Pt and Pd as a function of energy is displayed in Fig. 5(a). The $\sigma_{x y}^{S}$ at the $E_{F}$ is $2200(\hbar / \mathrm{e}) \mathrm{S} / \mathrm{cm}$ for $\mathrm{Pt}$ and $1242(\hbar / \mathrm{e}) \mathrm{S} / \mathrm{cm}$ for $\mathrm{Pd}$, being in good agreement with the corresponding results calculated previously using the linear muffin-tin orbital method with the atomic sphere approximation. 3, 31] We then evaluate numerically the energy derivative of the

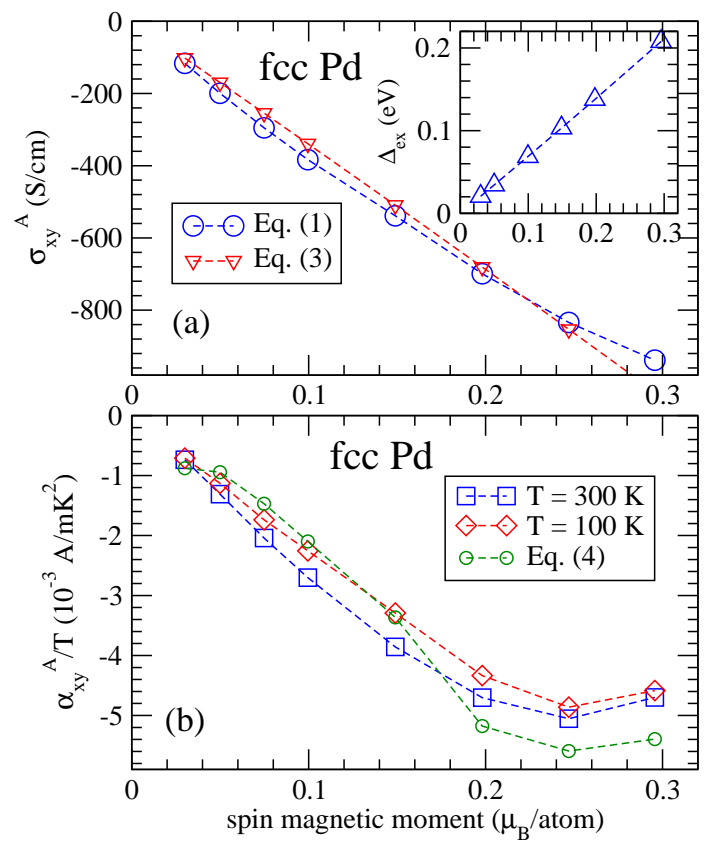

FIG. 4: (color online) (a) Anomalous Hall conductivity $\left(\sigma_{x y}^{A}\right)$ and (b) anomalous Nernst conductivity $\left(\alpha_{x y}^{A}\right)$ as a function of the induced spin magnetic moment $\left(m_{s}\right)$ in palladium. Exchange splitting $\left(\Delta_{e x}\right)$ is displayed as a function of $m_{s}$ in the inset in (a). In (b), $T$ denotes temperature.

SHC using the $\sigma_{x y}^{S}(E)$ displayed in Fig. 5. We obtain $\sigma_{x y}^{S}\left(E_{F}\right)^{\prime}=1081$ and $-4245(\hbar / \mathrm{e}) \mathrm{S} / \mathrm{cm}-\mathrm{eV}$ for $\mathrm{Pt}$ and $\mathrm{Pd}$, respectively. Figures $3(\mathrm{a})$ and $4(\mathrm{a})$ also show the $\sigma_{x y}^{A}$ evaluated using Eq. (3) together with the calculated $\sigma_{x y}^{S}\left(E_{F}\right)^{\prime}$ and $\Delta_{e x}$. It is clear that Eq. (3) holds very well for small $m_{s}$ up to $\sim 0.25 \mu_{B}$ /atom for Pt and $\mathrm{Pd}$ [Figs. 3(a) and 4(a)].

We have also calculated the $\mathrm{SHC}$ in the magnetized $\mathrm{Pt}$ and Pd metals. The calculated SHC for Pt and Pd is shown as a function of the spin magnetic moment in Fig. 5(b). In both $\mathrm{Pt}$ and $\mathrm{Pd}$, the SHC initially increases with $m_{s}$ up to $\sim 0.1 \mu_{B} /$ atom and then decreases slowly as $m_{s}$ further increases [Fig. 5(b)]. Nevertheless, the SHC for both $\mathrm{Pt}$ and $\mathrm{Pd}$ remains in the same order of magnitude all the way up to $m_{s}=0.5 \mu_{B}$ /atom.

The validity of Eq. (3) may be understood at the microscopic level. The two-current model can be derived from an approximation in which the spin-flipping part of the SOC is ignored. The spin-conserving part of the SOC can still lead to nontrivial results on the transverse transport coefficients. This non-flip approximation can be justified for crystals with inversion symmetry and in the limit of zero magnetization. This is because that Kramer's theorem implies a two-fold degeneracy of the band structure at general $k$-points even in the presence of the SOC. The SOC term in the Hamiltonian, being symmetric under spatial inversion and time reversal, must behave as a constant within the degenerate space. There- 

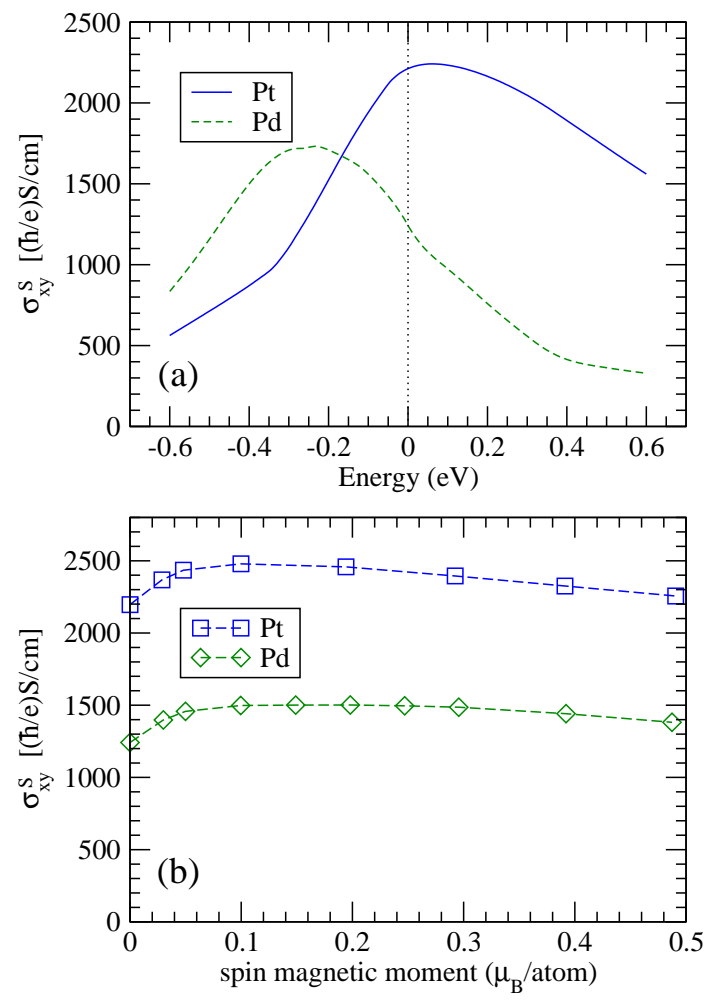

FIG. 5: (Color online) (a) Spin Hall conductivity $\left(\sigma_{x y}^{S}\right)$ as a function of energy in nonmagnetic $\mathrm{Pt}$ and $\mathrm{Pd}$ metals. The vertical dotted line at $0 \mathrm{eV}$ indicates the Fermi energy $\left(E_{F}\right)$. $\sigma_{x y}^{S}\left(E_{F}\right)=2200$ and $1242(\hbar / \mathrm{e}) \mathrm{S} / \mathrm{cm}$ for Pt and Pd, respectively. $\sigma_{x y}^{S}\left(E_{F}\right)^{\prime}=1081$ and $-4245(\hbar / \mathrm{e}) \mathrm{S} / \mathrm{cm}-\mathrm{eV}$ for Pt and $\mathrm{Pd}$, respectively. (b) Spin Hall conductivity as a function of the induced spin magnetic moment $\left(m_{s}\right)$ in magnetized $\mathrm{Pt}$ and Pd metals.

fore, it must also commute with the representation of the spin operator within the two-fold degenerate space. In the presence of a small magnetization, the degenerate bands are split to first order in the Zeeman energy according to the representation of the spin operator within each of the original degenerate space. Not being able to mix these split levels directly, the spin-flip part of the SOC term in the Hamiltonian can be safely discarded, because its residual effect must be of second order (in a process going out and back to the degenerate space).

\section{Anomalous Nernst effect}

Figures 3(b) and 4(b) indicate firstly that the anomalous Nernst conductivity $\alpha_{x y}^{A}$ increases monotonically with the spin moment $m_{s}$ for $m_{s}$ up to at least 0.5 $\mu_{B}$ /atom in $\mathrm{Pt}$ and for $m_{s}$ up to $0.25 \mu_{B}$ /atom in $\mathrm{Pd}$. Like $\sigma_{x y}^{A}, \alpha_{x y}^{A}$ is approximately proportional to $m_{s}$ for $m_{s} \leq \sim 0.20 \mu_{B}$ /atom in both Pt and Pd. Secondly, the calculated $\alpha_{x y}^{A}$ is large, especially in $\mathrm{Pt}$ [Fig. 3(b)]. In fact, $\alpha_{x y}^{A}$ for Pt at $m_{s} \geq 0.15 \mu_{B}$ /atom could be ten times larger than the intrinsic $\alpha_{x y}^{A}\left[\alpha_{x y}^{A} / \mathrm{T}=0.51 \times 10^{-3}\right.$ $\mathrm{A} /\left(\mathrm{m}-\mathrm{K}^{2}\right)$ at $\left.T=293 \mathrm{~K}\right]$ of iron [27]. The magnitude of $\alpha_{x y}^{A}$ for $\mathrm{Pd}$ for $m_{s} \geq 0.05 \mu_{B} /$ atom is also several times larger than that of iron[27].

At low temperatures, Eq. (2) can be simplified as the Mott relation,

$$
\alpha_{x y}^{A}=-\frac{\pi^{2}}{3} \frac{k_{B}^{2} T}{e} \sigma_{x y}^{A}(\mu)^{\prime},
$$

which relates the ANC to the AHC. Therefore, it is not surprising that the magnetized platinum has a very large $\alpha_{x y}^{A}$ since the $E_{F}$ is located on the steep slope of $\sigma_{x y}^{A}(E)$ [Fig. 1(b)], resulting in a large energy derivative of $\sigma_{x y}^{A}(E)$ at $E_{F}$. In Figs. 1(b) and 2(b), the $\alpha_{x y}^{A}$ calculated using the Mott relation [Eq. (4)] is displayed as a function of the induced $m_{s}$. Clearly, $\alpha_{x y}^{A}$ calculated directly $[$ Eq. (2)] and using the Mott relation at $T=100$ $\mathrm{K}$ are in good agreement with each other for Pt [see Fig. $3(\mathrm{~b})$ ] and also for $m_{s} \leq 0.15 \mu_{B}$ /atom for $\mathrm{Pd}$ [see Fig. 4(b)]. On the other hand, $\alpha_{x y}^{A}$ at $T=300 \mathrm{~K}$ calculated directly differs noticeably from that from the Mott relation, indicating that $T=300 \mathrm{~K}$ cannot be considered as a low temperature in this context.

Differentiating Eq. (3) and substituting the result into Eq. (4), we find

$$
\alpha_{x y}^{A} / T=-\frac{\pi^{2}}{3} \frac{k_{B}^{2} \Delta_{e x}}{\hbar} \sigma_{x y}^{s}(\mu)^{\prime \prime} .
$$

Eqs. (3) and (4) indicate that for small $m_{s}$, both the AHC and ANC are proportional to the exchangesplitting. As mentioned above, the exchange-splitting is almost a perfect linear function of $m_{s}$, and hence this explains why both the $\sigma_{x y}^{A}$ and $\alpha_{x y}^{A}$ are approximately proportional to $m_{s}$. Furthermore, this suggests that the $\sigma_{x y}^{A}$ and $\alpha_{x y}^{A}$ are proportional to each other for small $m_{s}$, as shown in Fig. 3 and Fig. 4. Therefore, we can rewrite Eq. (3) as

$$
\sigma_{x y}^{A}\left(E_{F}\right) \approx\left[\frac{e}{\hbar} \frac{\Delta_{e x}\left(m_{s}^{0}\right)}{m_{s}^{0}} \sigma_{x y}^{S}\left(E_{F}\right)^{\prime}\right] m_{s}=\beta m_{s},
$$

where constant $\beta$ can be determined solely by firstprinciple calculations for a certain spin moment $m_{s}^{0}$. In the present work, we find that $\beta=788 \mathrm{~S} / \mathrm{cm} / \mu_{B}$ for $\mathrm{Pt}$ and $\beta=-2921 \mathrm{~S} / \mathrm{cm} / \mu_{B}$ for $\mathrm{Pd}$. Similarly, we can rewrite Eq. (4) as

$$
\alpha_{x y}^{A} / T \approx-\left[\frac{\pi^{2}}{3} \frac{k_{B}^{2} \Delta_{e x}\left(m_{s}^{0}\right)}{\hbar m_{s}^{0}} \sigma_{x y}^{S}\left(E_{F}\right)^{\prime \prime}\right] m_{s}=\gamma m_{s} .
$$

And using the results of the first-principle calculations, we obtain that constant $\gamma=0.034 \mathrm{~A} /\left(\mathrm{m}-\mathrm{K}^{2} \mu_{B}\right)$ for $\mathrm{Pt}$ and $\gamma=-0.027 \mathrm{~A} /\left(\mathrm{m}-\mathrm{K}^{2} \mu_{B}\right)$ for $\mathrm{Pd}$.

\section{CLOSING REMARKS}

Recently, the possible magnetic proximity-induced spin moment in $\mathrm{Pt}$ films in the $\mathrm{Pt} / \mathrm{YIG}$ bilayers 
was measured by magnetic x-ray circular dichroism experiments [4], and $m_{s}$ was found to be $0.054 \mu_{B}$ /atom at $300 \mathrm{~K}$ and $0.076 \mu_{B}$ /atom at $20 \mathrm{~K}$. Using $m_{s}=0.05$ $\mu_{B}$ /atom together with Eqs. (6) and (7), we can estimate the intrinsic AHC and ANC for the Pt film to be $\sigma_{x y}^{A}=40 \mathrm{~S} / \mathrm{cm}$ and $\alpha_{x y}^{A}=0.51 \mathrm{~A} /\left(\mathrm{m}-\mathrm{K}^{2}\right)(T=300$ $\mathrm{K})$. The anomalous Seebeck coefficient $E_{y} /\left(-\partial_{x} T\right)=$ $\rho_{x x}\left(\alpha_{x y}-S \sigma_{x y}\right)$ where $S=\alpha_{x x} / \sigma_{x x}$ is the ordinary Seebeck coefficient. At $T=300 \mathrm{~K}, \rho_{x x}=10.8 \mu \Omega \mathrm{cm}$ and $S=-11.28 \mu \mathrm{V} / \mathrm{K}$ (see Refs. 45 and 46). Resultantly, $E_{y} /\left(-\partial_{x} T\right)=0.058 \mu \mathrm{V} / \mathrm{K}$. Using the sample sizes and the temperature gradient in the Pt/YIG bilayers [47, 48], one would obtain the Hall voltage due to the ANE in the order of $\sim 0.1 \mu \mathrm{V}$, being comparable with the Hall voltage $(\sim 0.1 \mu \mathrm{V}$ in $\mathrm{Au} / \mathrm{YIG}$ and $\sim 1.0 \mu \mathrm{V}$ in Pt/YIG) produced by the spin Seebeck effect via the inverse spin Hall effect.

\section{Acknowledgments}

G.Y.G. acknowledges support from the Ministry of Science and Technology, the Academia Sinica Thematic Research Program and NCTS of Taiwan, and thanks ShangFan Lee and Shiming Zhou for stimulating discussions. G.Y.G. also acknowledges partial support from NBRPC (No. 2012CB921300 and No. 2013CB921900), and NSFC (No. 91121004) during his visit at Peking University. Q.N. was supported in part by DOE-DMSE (No. DEFG03-02ER45958) and the Welch Foundation (No. F$1255)$.

* Electronic address: gyguo@phys.ntu.edu.tw

[1] G. A. Prinz, Science 282, 1660 (1998); S. A. Wolf, D. D. Awschalom, R. Chtchelkanova, and D. M. Treger, ibid., 294, 1488 (2001)

[2] I. Zutic, J. Fabianm and S. D. Sarma, Rev. Mod. Phys. 76, 323 (2004).

[3] G. Y. Guo, S. Murakami, T.-W. Chen, and N. Nagaosa, Phys. Rev. Lett. 100, 096401 (2008).

[4] Z. Feng, J. Hu, L. Sun, B. You, D. Wu, J. Du, W. Zhang, A. Hu, Y. Yang, D. M. Tang, B. S. Zhang, and H. F. Ding, Phys. Rev. B 85, 214423 (2012).

[5] A. Hoffman, IEEE Trans. Mag. 49, 5172 (2013).

[6] S. O. Valenzuela, M. Tinkham, Nature 442, 176 (2006).

[7] E. Saitoh, M. Ueda, H. Miyajima, and G. Tatara, Appl. Phys. Lett. 88, 182509 (2006).

[8] K. Uchida, S. Takahashi, K. Harii, J. Ieda, W. Koshibae, K. Ando, S. Maekawa, and E. Saitoh, Nature 455, 778 (2008).

[9] Y. Kajiwara, K. Harii, S. Takahashi, J. Ohe, K. Uchida, M. Mizuguchi, H. Umezawa, H. Kawai, K. Ando, K. Takanashi, S. Maekawa, and E. Saitoh, Nature 464, 262 (2010).

[10] I. M. Miron, K. Garello, G. Gaudin, P.-J. Zermatten, M. V. Costache, S. Auffret, S. Bandiera, B. Rodmacq, A.
Schuhl and P. Gambardella, Nature 476, 189 (2011).

[11] Wilhelm, Phys. Rev. Lett. 85, 413 (2000).

[12] Antel, Phys. Rev. B 60, 12933 (1999).

[13] S. Blügel, Phys. Rev. B 51, 2025 (1995).

[14] A. Smogunov, A. Dal Corso, A. Delin, R. Weht, and E. Tosatti, Nat. Nanotechnol. 3, 22 (2008).

[15] J.-C. Tung and G. Y. Guo, Phys. Rev. B 81, 094422 (2010).

[16] N. Nagaosa, J. Sinova, S. Onoda, A. H. MacDonald, and N. P. Ong, Rev. Mod. Phys. 82, 1539 (2010)

[17] E. H. Hall, Phil. Mag. B 12, 157 (1881).

[18] S. Y. Huang, X. Fan, D. Qu, Y. P. Chen, W. G. Wang, J. Wu, T. Y. Chen, J. Q. Xiao, and C. L. Chien, Phys. Rev. Lett. 109, 107204 (2012).

[19] W. Nernst, Ann. Phys. 267, 760 (1887).

[20] Z. A. Xu, N. P. Ong, Y. Wang, T. Kakeshita, and S. Uchida, Nature 406, 486 (2000).

[21] W.-L. Lee, S. Watauchi, V. L. Miller, R. J. Cava, and N. P. Ong, Phys. Rev. Lett. 93, 226601 (2004).

[22] D. Xiao, Y. Yao, Z. Fang and Q. Niu, Phys. Rev. Lett. 97, 026603 (2006).

[23] T. Miyasato, N. Abe, T. Fujii, A. Asamitsu, S. Onoda, Y. Onose, N. Nagaosa, and Y. Tokura, Phys. Rev. Lett. 99, 086602 (2007).

[24] D. L. Bergman and V. Oganesyan, Phys. Rev. Lett. 104, 066601 (2010).

[25] T. Yokoyama and S. Murakami, Phys. Rev. B 837, 161407 (2011).

[26] S. Y. Huang, W. G. Wang, S. F. Lee, J. Kwo, and C. L. Chien, Phys. Rev. Lett. 107, 216604 (2011).

[27] J. Weischenberg, F. Freimuth, S. Blügel, and Y. Mokrousov, Phys. Rev. B 87, 060406 (2013).

[28] M. Schmid, S. Srichandan, D. Meier, T. Kuschel, J.-M. Schmalhorst, M. Vogel, G. Reiss, C. Strunk, and C. H. Back, Phys. Rev. Lett. 111, 187201 (2013).

[29] K. Uchida, Nature Mater. 9, 894 (2010).

[30] C. M. Jaworski, J. Yang, S. Mack, D. D. Awschalom, J. P. Heremans, and R. C. Myers, Nature Mater. 9, 898 (2010).

[31] G. Y. Guo, J. Appl. Phys. 105, 07 C701 (2009).

[32] R. Huguenin, G. P. Pells, and D. N. Baldock, J. Phys. F: Metal Phys. 1, 281 (1971).

[33] Z. Celinski, et al., Phys. Rev. Lett. 65, 1156 (1990).

[34] J. R. Childress, et al., J. Magn. Magn. Mater. 130, L25 (1994).

[35] X. Zhou, L. Ma, Z. Shi, and S. M. Zhou, arXiv:1309.4841 (2013).

[36] D. Xiao, M.-C. Chang, and Q. Niu, Rev. Mod. Phys. 82, 1959 (2010)

[37] Y. Yao et al., Phys. Rev. Lett. 92,037204 (2004).

[38] S.H. Vosko, L. Wilk, and M. Nusair, Can. J. Phys. 58, 1200 (1980).

[39] P. Blaha, K. Schwarz, G. Madsen, D.Kvasnicka, and J. Luitz, WIEN2K, An Augmented Plane Wave Local Orbitals Program for Calculating Crystal Properties (Technische University Wien, Austria, 2002)

[40] O. Jepson and O. K. Anderson, Solid State Commun. 9, 1763 (1971)

[41] J.-C. Tung, H.-R. Fuh, and G. Y. Guo, Phys. Rev. B 86, 024435 (2012).

[42] J.-C. Tung, and G. Y. Guo, New J. Phys. 15, 033014 (2013).

[43] Note that in Refs. 41 and 42, $2 \frac{\hbar}{e} \sigma_{x y}^{S}=\sigma_{x y}^{\uparrow}-\sigma_{x y}^{\downarrow}$ in 
Eq. (5) and Eq. (2b), respectively, should be $-2 \frac{\hbar}{e} \sigma_{x y}^{S}=$ $\sigma_{x y}^{\uparrow}-\sigma_{x y}^{\downarrow}$. Consequently, the signs of the spin-up and spin-down Hall conductivities $\left(\sigma_{x y}^{\uparrow}\right.$ and $\left.\sigma_{x y}^{\downarrow}\right)$ as well as the spin polarization of the Hall current $\left(P^{H}\right)$ listed in Tables I and II in Refs. 41 and 42 should be reversed.

[44] Y. M. Lu, Y. Choi, C. M. Ortega, X. M. Cheng, J. W. Cai, S. Y. Huang, L. Sun, and C. L. Chien, Phys. Rev. Lett. 111,147207 (2013).

[45] Landolt-Börnstein, New Series, Volume 15, Metals: Electronic Transport Phenomena, Subvolume a by J. Bass and K. H. Fischer (Springer-Verlag, Berlin, 1982).

[46] Landolt-Börnstein, New Series, Volume 15, Metals: Electronic Transport Phenomena, Subvolume b by J. Bass, J. S. Dugdale, C. L. Foiles and A. Myers (Springer-Verlag, Berlin, 1982).

[47] D. Qu, S. Y. Huang, J. Hu, R. Wu, and C. L. Chien, Phys. Rev. Lett. 110,067206 (2013).

[48] T. Kikkawa, K. Uchida, Y. Shiomi, Z. Qiu, D. Hou, D. Tian, H. Nakayama, X.-F. Jin, and E. Saitoh, Phys. Rev. Lett. 110,067207 (2013). 